\title{
A study on Semantic Association Ellipsis on the View of ICM by Computer Technology
}

\author{
YANG ZhiShang ${ }^{1, \text { a }}$ \\ ${ }^{1}$ Changsha Medical University, Changsha, Hunan,413000 China \\ a284204813@qq.com
}

Keywords: Semantic association; Ellipsis; ICM

\begin{abstract}
As a special kind of linguistic phenomenon and bridging ties, Semantic association ellipsis is a kind of semantic vacancy, omitted contents can't be recovered through context, the understanding of ellipsis is a difficulty in the study on semantic association ellipsis. This paper which based on the ICM theory, discussed the explanatory power of ICM to semantic association ellipsis around the experience, openness, relevance and other characteristics.
\end{abstract}

\section{Introduction}

Ellipsis is a major feature of language. As a special linguistic phenomenon and cohesive link, semantic association ellipsis in discourse is sometimes a lack of semantic meaning and can not be found out from the context, which has been a problem in studies of ellipsis. Default information can only be added by semantically associating some of the undefined knowledge in the cognitive world (ICM and background). Bally, the student of Saussure's, proposed the notion of semantic association in 1909, which refers to the semantic connection established between the cognitive subject and the existing information based in the mind when it accepts the foreign language messages, and it is the medium that connects external language with internal concept, is a form of discourse understanding based on semantics, taking psychological as medium and culture as a background, which directly influences the objectivity and correctness of the cognitive subject in understanding the discourse. The omissions in the discourse can be activated by the cognitive world (ICM and background) through association of semantics, recalling many words that may fill in the semantic gap, as well as past experiences, extensive knowledge of natural, social and cultural contexts; we can do semantic coding, associating and predicting the omitted information, to comprehend the intents expressed by the author or the speaker. This article try to interpret the semantic association ellipsis from the view of the ICM theory and background knowledge of cognitive linguistics.

\section{ICM and semantic associative ellipsis}

\section{A. Overview of ICM}

ICM(“Idealized Cognitive Model”) is a theory of cognitive theory proposed by Lakoff in 1987. Model refers to the cognitive structures and frameworks that are relatively stable to a particular event or concept. Cognitive Model(CM) is the specific way for people to obtain acknowledges, is the psychological representation of cognitive experience in a specific cultural context formed on the basis of repeated interactions with the objective worlds, that means the specific way in which our knowledge is organized and characterized as a "field" of relevant knowledge and experience. The so-called ICM refers to abstract, unified and idealistic understanding made by speakers in a particular cultural context to the experience and knowledge in a certain field, which is a complex and integrated conclusion based on many CM Type structures, and is a complex cognitive model $\mathrm{j}$ with the properties of a Gestalt. From the cognitive point of view, it can be expressed as: 


\section{$\mathrm{ICM}=\mathrm{CM} 1+\mathrm{CM} 2+\mathrm{CM} 3 \cdots \mathrm{CMn}$ 。}

\section{B Semantic associative ellipsis in the context of ICM}

Cognitive world knowledge, by popularity, representativeness and idealization, can be divided into: ICM and background knowledge. When people understand the discourse, they tend to use the knowledge in the cognitive world to "bypass" the relevant information in the sentence, and continuously proactively create coherence. Through the information provided in the sentences, the anaphoric relations between the concepts can be activated and the semantic associations of words can be built, and then they can form a common sense of the cognitive world, or we can say that looking for a concept of meaning to establish a unified topic, so that you can get the coherence of the discourse, also can understand the discourse information. Ellipsis is a common phenomenon in language, which exists in all forms of discourses. It is impossible for people to have a complete list of what they want to say in the practical application of language. They often make some choices in the overall information and use language to express, so the language of communication is always passed more than its literal meaning with much more information, which is the principle of language communicative economy. If the default information can not be found out from the context, it will cause the fracture of the bridging bond. Only through the semantic association can we understand the default information. But how does the ICM explain semantic association ellipsis? Readers or listeners can take the initiative to construct a cognitive world model (ICM) in the process of discourse comprehension relying on the background knowledge, and can obtain the relevant information activated by some components in the sentences by associating, selecting the appropriate knowledge to fill the default information in order to obtain the overall meaning of discourse.

\section{The role of metaphor in the construction of the topic}

Aristotle believes that man is a rational animal. As a kind of rhetorical act of persuading and inducing cooperation, metaphor must also be a form of "serving people with reason". The important manifestation of metaphorical function of "serving people with reason" is that it has the function of constructing an argument. Metaphor not only provides a way to recognize the world, but also play an important role in the argument, mainly in two aspects: (a) Metaphor itself constitutes a topic, and (b) metaphor is an important part of the topic. First, the metaphor itself constitutes an independent topic. It puts two different things "in dislocation" together, forcing the listener to discover or create some sort of similarity between the two. If the social and cultural backgrounds of rhetoricians and listeners are the same or similar, the similarities between the two are not hard to find or to create. In this case, the meaning of the metaphor will be "filtered" or "screened" under the guidance or restriction of the subject framework and be collocated with the reference terms. Once the similarities between the two are established, subject $\mathrm{A}$ and the relational equation of the reference $\mathrm{B}$ is established: Subject $\mathrm{A}=$ Reference B. Therefore, similarity has become a bridge connecting the two. From the point of view of the topic, the subject and the reference are the "materials" of the thesis, and the relational equation of subject and reference is $\mathrm{A}=\mathrm{B}$, which is the "proposition" of the thesis, while the similarities found or created by the audience between the subject and the metaphor is the "reason."

Quantitative analysis of the corpus are researched, and categories or morpheme in the four-character acronyms are mainly verbs, nouns, adjectives, adverbs and tone words, and the statistical results are in Table 1.

Table 1 Statistical table of part of speech

\begin{tabular}{|c|c|c|c|c|c|}
\hline $\begin{array}{c}\text { Part of } \\
\text { speech }\end{array}$ & Verb & Noun & Adjective & Adverb & $\begin{array}{c}\text { Function } \\
\text { word }\end{array}$ \\
\hline Proportion & $38 \%$ & $27 \%$ & $21 \%$ & $10 \%$ & $4 \%$ \\
\hline
\end{tabular}


Syntactic rules appear as acronyms words or morphemes where the words in the upper grammatical units - phrases or sentences in the syntactic composition of the main predicates, head nouns, adverbials, subjects and attribute languages, and the statistical results are in Table 2.

Table 2 Statistical table of syntactic components

\begin{tabular}{|c|l|l|l|l|c|}
\hline $\begin{array}{c}\text { Syntactic } \\
\text { components }\end{array}$ & Predicate & Head noun & Adverbial & Subject & Adnex \\
\hline Proportion & $27 \%$ & $20 \%$ & $14 \%$ & $13 \%$ & $9 \%$ \\
\hline
\end{tabular}

The reason is mainly because the verbs or adjectives used as predicate or central language are usually the core of the syntactic structure and semantic interpretation. Without it, the meaning of the sentence is loosened. The framework is ambiguous and versatile as a way of understanding social, linguistic and cultural connections. From the perspective of the functional orientation of the framework, the framework's effect is manifested in that when the framework of two logically equivalent arguments on the same issue affects the individuals to make different choices, the effect is mostly reflected in the influence of discourse content. This "mid-level" orientation helped the media study distinguish between social truth and media truth. From the perspective of the dissemination of language, language itself is a medium as one of the important carriers of information to be carried and disseminated. Therefore, in the linguistic context, the analytical framework of social truth and media truth(specifically, language truth) are applied equally. The unity of the individual, the language and the society in the study of linguistics is scientific and consistent with the law of historical development. The history of the development of language has verified this point, and it is crucial to pay attention to the social function of language and the communication and psychological mechanism of individual.

Therefore, although the four example sentences are both in the form of "Tao + AC," there are different types in the semantic grid. It should be said that different levels of framework classification also provide more detailed classification criteria for understanding semantics.

\begin{tabular}{|c|c|c|c|}
\hline \multicolumn{2}{|c}{ Table 3 Verb-object construction “Tao + AC” } \\
construction & $\begin{array}{c}\text { Grammatical } \\
\text { meaning }\end{array}$ & $\begin{array}{c}\text { Discourse } \\
\text { construction }\end{array}$ & Frame type \\
\hline $\begin{array}{c}\text { Tao Shenghuo(Seek } \\
\text { living) }\end{array}$ & $\begin{array}{c}\text { Object represents } \\
\text { tools }\end{array}$ & filter & $\begin{array}{c}\text { Interactional framework; } \\
\text { Social framework }\end{array}$ \\
\hline $\begin{array}{c}\text { Tao } \\
\text { Shuofa(confront) }\end{array}$ & $\begin{array}{c}\text { Object represents } \\
\text { object }\end{array}$ & filter & $\begin{array}{c}\text { Cognitive framework; Social } \\
\text { framework }\end{array}$ \\
\hline $\begin{array}{c}\text { Tao Laopo(To get } \\
\text { married) }\end{array}$ & $\begin{array}{c}\text { Object represents } \\
\text { object }\end{array}$ & filter & $\begin{array}{c}\text { Cognitive framework; Social } \\
\text { framework }\end{array}$ \\
\hline $\begin{array}{c}\text { Tao Huanxin(To } \\
\text { please someone) }\end{array}$ & $\begin{array}{c}\text { Object represents } \\
\text { benifit }\end{array}$ & construct & $\begin{array}{c}\text { Interactional framework; } \\
\text { Social framework }\end{array}$ \\
\hline $\begin{array}{c}\text { Tao Gongdao(Seek } \\
\text { Justice) }\end{array}$ & $\begin{array}{c}\text { Object represents } \\
\text { benifit }\end{array}$ & construct & $\begin{array}{c}\text { Cognitive framework; Social } \\
\text { framework }\end{array}$ \\
\hline $\begin{array}{c}\text { Tao Meiqu(Get an } \\
\text { embarrassing rebuff) }\end{array}$ & $\begin{array}{c}\text { Object represents } \\
\text { state }\end{array}$ & construct & $\begin{array}{c}\text { Cognitive framework; Social } \\
\text { framework }\end{array}$ \\
\hline
\end{tabular}


Grammatical structure's incomplete will lead to ambiguous, and the following will discuss Chinese ambiguous sentences caused by the semantic association ellipsis.

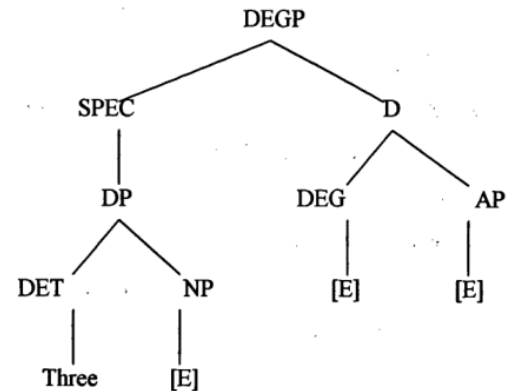

Figure 4 Structure 1

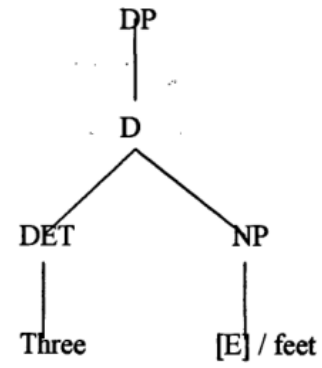

Figure 5 Structure 2

"There are two goals of cognitive science, one of which is interpretive, aiming to establish a set of theories through the study of human cognitive activity to explain the various aspects of cognition, which are then put into experiments and computer simulations test."(Gardenfors 1996: 159). From a cognitive perspective to study the language, we can use the method of construction mode or theory to analyze the processing of language information to explain certain linguistic phenomena. As it is shown in Figure 6 below:

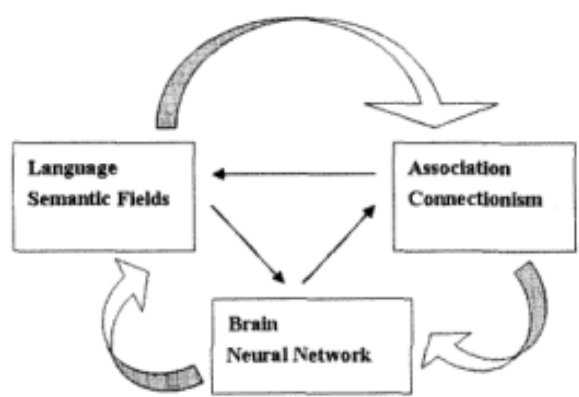

Figure 6 Cognitive model of semantic association ellipsis

The figure is a cognitive cycle model, which is two-way, and it can be counter-clockwise cycle or clockwise cycle. Clockwise cycle interpretation: Language semantic fields - Association connectionism - Brain neural network, starting from the language semantic fields, the vocabulary network of the semantic field gives different paths of association, which provide an effective search range to recall the omitted content; People's associative model of cognition carries out parallel processing and processing on all possible associative terms in the semantic field, forming output units at the output layers and filling the most appropriate and accurate vocabularies as omissions. enseignement



Figure 7 the vocabulary network of semantic association filed

In figure 7 , we take enseignement as the center to form four lines radiating to four different paths. The first line from left is associated with the same roots; The second line from left is associated with the semantic correlation; The third line from left is associated with the same suffix; The fourth line from left is associated with the same pronunciation. These associations are multi-directional, multi-angle, multi-level infinite expansion to the surroundings. 


\section{Conclusion}

The understanding of semantic association ellipsis on the view of ICM is a complex cognitive activity. In this dissertation, the concept of ICM, semantic association ellipsis on the view of ICM and the relations between ICM features and semantic association ellipsis are elaborated in details. It is proposed that for disclosures with cognitive semantic association ellipsis, cognitive subjects should take more cognitive actions to activate corresponding knowledge of the world(ICM and background knowledge), in order to add the default information with association and obtain the psychological coherence to understand.

\section{References}

[1] Xu Hui. The classification of Ellipsis and Relevant Cognitive Methods on Ellipsis[J].Journal of Xihua University(Philosophy\&Social Science),2017,01:99-103.

[2] Yang Leida, Zhao Genglin. The Cognitive Model of Semantic Association Ellipsis[J].Foreign Language Education,2013,04:37-40.

[3] Yang Leida, Zhao Genglin. Cognitive Network Connection Mode of Ellipsis-The Thinking triggered by a telegram[J].Foreign Language Research,2013,05:45-50.

[4]Zeng Ruiming, Long Yanhui. Study on Chinese Ambiguity Sentences Caused by Semantic Associative Ellipsis[J].Journal of Human Institute of Humanities, Science and Technology,2010,01:91-92.

[5]Yang Zhishang. The understanding of Ellipsis in Listening Discourses Based on the ICM Theory[J].Journal of Hebei Polytechnic University(Social Science Edition),2010,05:180-182. 